\title{
Electroencephalographic coherences during emotion identification task
}

\author{
Erzsébet Marosi Holczberger*, Jorge Bernal, Juan Silva, Guillermina Yañez, Mario Rodríguez, \\ Belén Prieto, Vicente Guerrero
}

National University of Mexico, FES Iztacala, Neurosciences Department, Mexico City, Mexico

Email: "

Received 11 August 2012; revised 16 September 2012; accepted 26 September 2012

\begin{abstract}
Coherence, a measure of spectral similarity, may estimate cortical coupling between two EEG signals as a function of frequency. The coherence between EEG signals from different brain areas depends on the structural connection and functional coupling between two regions. The theory of hemispheric specialization proposes left hemisphere activation to positive emotions and right one to negative emotions. Other proposal is that right hemisphere activation occurs with emotional stimuli. In the present study EEG coherences were calculated during the presentation of the International Affective Pictures to 36 healthy male university students. The subjects' task was to determine the valence of the stimuli. Base line recording were done with subjects observing the blank monitor. Our results showed higher coherences during the emotional condition. No differences between negative and positive emotions were obtained. Neutral pictures evoked the highest coherence values. These data suggest that coherences between functionally coupled brain areas do not reflect emotional recognition of the valence of stimuli, but they are internal indicators of different mental processes such as memory, mental effort and arousal. Our findings do not support the theory of hemispheric specialization, neither the theory of right hemisphere; rather they suggest a complex activation pattern that involves mostly frontal areas in connection with the entire cortex.
\end{abstract}

Keywords: EEG Coherences; Emotions of Positive and Negative Valence; International Affective Pictures

\section{INTRODUCTION}

Different brain areas process various aspects of information, as perception and recognition of positive and negative emotions, in parallel. The way how is this emo-

"Corresponding author. tional information integrated into perception or action is not known. However, we know that different cortical (frontal, parietal, occipital and temporal) and subcortical areas (especially the entire limbic system) participate in its production. Coherence, a measure of spectral similarity, may estimate cortical coupling, a correlation between two EEG signals as a function of frequency, hence it provides a method for identifying frequency bands that contain inter-channel synchronization [1]. The coherence between EEG signals from different brain areas depends on the structural connection and functional coupling between these regions [2]. Usually in EEG recordings the coherence is high between close electrodes and falls dramatically with the growth of interelectrode distance that may be explained by volume conduction. Nevertheless, if rhythmic activities dominate in EEG, the degree of cooperativity increases in a very significant way, and coherent activity can occur over larger extents of the cortical surface [3]. In some cases the dependence of coherence on distance may be not gradual: high coherence between spatially separated cortical areas is registered although their coherence with intermediate sites is low [4,5]. This evidences the fact that the EEG rhythms synchronization is not a result of direct volume conduction but is related to interaction of distant areas that participate in mutual functioning.

Coherence analysis seems to be suitable for studying brain functions, because it measures a possible mechanism of integration. It offers promise in revealing neuronal correlates governing specific mental functions, as emotions among others.

The literature about emotion is replete with different proposals regarding the interrelations among different forms of positive and negative affect. Various theories have been proposed on, but none have succeeded in explaining more than a small part of empirical data. For example, Russell [6] proposed that there exists a single bivalent dimension that ranges from unpleasant to pleasant affect, with a second dimension that reflects arousal. This theory suggests that valence and intensity may ex- 
plain the identification of the different affective stimuli.

In contrast, others sustain that different cortical areas (left and right frontal cortex) show special patters of brain electric activity reflecting differences in approach vs. withdrawal, or positive vs. negative emotions [7].

Coherence values are a convenient method to study emotion; nevertheless, there are few studies that relate EEG coherences to emotional valence identification. A study of Bos [8] reported that positive emotions resulted in higher frontal coherence in alpha, and higher right parietal beta, as compared to negative emotion.

Miskovic \& Miskovic [9] examined coherences during affective image presentation and found an increase in beta $(14-40 \mathrm{~Hz})$ and decrease in theta coherences. In the same sense, Güntekin \& Basar [10] also reported higher beta response for unpleasant than for pleasant and neutral pictures.

Putman [11] calculated correlation between slow (1 - 3 $\mathrm{Hz})$ and fast $(13-30 \mathrm{~Hz})$ activity ( $\delta$ - $\beta$ correlation) in order to find functional synchronization between limbic and cortical brain systems. The author states that deltabeta coherence may reflect emotion regulation processes and suggests that $\delta$ - $\beta$ correlation may be a useful tool in the experimental study of affective processes. His results showed a negative association between $\delta$ - $\beta$ coherence and in self-reported trait anxiety and attentional threatavoidance, but no association with behavioral inhibition was found.

Sutton et al. [12] carried out a PET study during the presentation of aversive or appetitive pictures. The authors reported significant left-sided metabolic increase to appetitive pictures in inferior and superior prefrontal cortex, premotor and motor regions and nucleus accumbens and to aversive pictures in the right prefrontal cortex and amygdala.

Meanwhile, Wager et al. [13] in their meta-analysis examined 55 publications (43 PET and $12 \mathrm{fMRI}$ ) in search of specific regions associated with emotional activation in general, with specific emotions and different induction methods. In contrast with Sutton et al. the authors did not report valence related hemisphere specialization. On the contrary, their revision yielded as results that left prefrontal cortex apparently has a general role in emotional processing across all categories and domains of interest, and temporal, parietal and occipital cortices (as well as limbic areas) also show important activations.

As both theories and experimental results are often conflicting, the aim of this paper is to examine and describe in detail the topography and frequency structure of EEG coherences during valence identification of positive and negative images having as control situation the presentation of neutral pictures and baseline recording (open eye condition without task).
Based on the meta-analysis made by Wager et al. [13] we expect significant differences between emotional conditions at correlations between left prefrontal cortex and other cortical areas without hemispheric specialization according to valence.

\section{EXPERIMENTAL PROCEDURE}

\subsection{Participants}

The sample consisted of 36 healthy male volunteers within the age range of 20 - 25 years (mean age 22.31 years). Only male subjects were selected due to gender determined EEG differences reported by various authors [14-16]. All participants were right-handed, with normal or corrected-to normal vision and were not under prescription medication. Subjects with head injury, history of nervous system diseases, convulsive disorders or abnormal EEG with paroxistic activity do not participated in this experiment. The experimental procedure was conducted in accordance with the Declaration of Helsinki and was carried out with the adequate understanding and written consent of the subjects.

\subsection{Stimuli}

Three hundred previously validated images of the "International Affective Pictures” series were presented in a fully random order on the screen of a computer. Hundred of them were of positive valence (pleasant ones), the other hundred of negative valence (unpleasant ones) and 100 were neutral.

Provided that subject's judgment on emotional content of the stimuli is widely variable and the original validation was carried out with subjects of different cultural background, the picture series were categorized for the Mexican college population that took place with 40 young male subjects (others than the experimental subjects) and in this study we included only those pictures that had at least $80 \%$ of coincidence in judging the emotional valence of the images.

\subsection{Procedure}

Subjects sat $80 \mathrm{~cm}$ from the monitor where the pictures appeared. Each trial started with a fixation point (a cross) in the centre of the screen during $200 \mathrm{~ms}$. Then the image was presented during $2000 \mathrm{~ms}$. When it disappeared a question mark asked for response. The subjects' task was to determine the valence of the stimuli and to answer pressing " 1 " key on the number pad if the image was pleasant, the " 2 " key if it was unpleasant and the " 3 ” key if it was neutral. The response interval lasted until the participant responded, upon which a new trial began. The participants were instructed that the speed of the re- 
sponses was not important for the experiment and that they were welcome to take a small break, to blink or to adjust a more comfortable position, when the pictures were not present. Subjects were asked to avoid eye movement, withhold blinking or movements while the pictures were on the screen.

At the beginning of every session, a 5 minutes baseline EEG was recorded, with subjects watching the blank screen of the computer.

\subsection{EEG Recording}

Each subject wore a fitted electrode cap using the 21channel montage as specified according to the $10-20$ International system (FP1, FP2, FPZ, F7, F3, FZ, F4, F8, T7, C3, CZ, C4, T8, P7, P3, PZ, P4, P8, O1, OZ, O2) with linked earlobes as reference. Electrode impedances were always maintained below $5 \mathrm{~K}$ ohms. Electrooculogram (EOG) was recorded from electrodes placed supraorbitally at the outer canthus of the eye. Data acquisition was continuous with a sampling rate of $512 \mathrm{~Hz}$. The filter was set to $70 \mathrm{~Hz}$ with a bandwidth of $1.5 \mathrm{~Hz}-30 \mathrm{~Hz}$. EEG and EOG signals were amplified with a Neuroscan SynAmps System. The segmentation and averaging of EEG signals were performed using $\mathrm{Scan}^{\mathrm{TM}} 4.3$ software. The continuous EEG recordings were segmented in epochs of 1275 ms beginning with the stimulus onset. Segments containing eye blinks and artifacts were on and off-line rejected. At least 1 to 2 minutes of clean EEG was subjected to the Fast Fourier Transform. Band limits were defined as follows: delta $(1.75-3.91 \mathrm{~Hz})$, theta $(3.91$ $7.81 \mathrm{~Hz})$, alpha $(7.81-12.5 \mathrm{~Hz})$ and beta $(12.5-24.22$ $\mathrm{Hz})$. The ongoing EEG tracings were constantly monitored during the experiment to keep the quality of recording and the subject's wakefulness level under steady watch.

Segments with erroneous identification of valence of the stimuli and subjects that had small number of correct and artifact free responses were eliminated from this study.

\subsection{Statistical Analysis}

After cleaning the EEG, coherence spectra values were calculated between all possible channel pairs on the scalp.

Then, four series of repeated-measures ANOVA were performed separately for the four frequency bands between the four conditions for every possible combination of the 21 electrodes (Statistica 8). Fisher's Least Square Differences test was used as post-hoc analyses. Repeated calculation for each one of the four bands of rmANOVA is prone to Type I error therefore in the present paper the results will be reported only when it attains statistical significance higher than 0.01 .

\section{RESULTS}

Behavioral data showed that negative stimuli elicited $93 \%$ of hits, positive $70 \%$ of hits and neutral stimuli only $63 \%$ as they were often confounded with positive images. Given that we had to eliminate higher number of EEG segments to neutral stimuli, we assume higher difficulty in its identification.

Significant differences in coherence values between positive (1) and negative (2) picture presentations were negligible, as the significance level of the results did not reach the required $\mathrm{p}<0.01$ level.

Significant differences between EEG coherence to positive (1) and neutral (3) images are shown in Table 1. We can estimate that neutral pictures elicited higher coherences than positive ones, in all bands. We have obtained more significant differences in the beta band. The interconnections of left prefrontal cortex were the most frequent, however in some cases midline and right prefrontal, frontotemporal connections with central, parietal, temporal and occipital cortices were also significant.

Table 2 shows the significant differences in coherence values between negative (2) and neutral (3) picture presentations. We can see that negative pictures elicited lower coherence values, than neutral ones, in all bands, similar to positive pictures, however in less abundant connections. In this case, the alpha band had more significant results in frontal, temporal, frontotemporal and parietal interconnections.

Comparing baseline recordings with emotional conditions ( 1 vs 4 , 2 vs 4 and 3 vs 4) nearly all comparisons were highly significant having generally baseline values with lower coherences than emotional or neutral conditions, with very few exceptions, in delta and alpha bands. In beta band there were no exceptions. The situation was different in the theta band where baseline recording had higher coherence values, than responses to positive and negative picture presentations.

\section{DISCUSSION}

EEG coherence, being the covariance of spectral activity at two electrode sites, is a measure of synchrony of neuronal activity and thus can be used as an indicator of effective cortical connectivity. The induction of an intentional act is accompanied by changes in brain state and consequently in EEG recordings. In general, we could observe that when subjects had no task to accomplish (watching the blank screen), the coherence values were lower (with some negligible exceptions). This is a completely understandable finding, as any task requires more collaboration of brain structures than more passive states. However, this relation was reversed in the theta band where positive and negative images evoked lower coherences values than baseline recording. Therefore, we 
Table 1. Significant differences in coherence values between positive (1) and neutral (3) picture presentations. (Asterisk marks significance level $\mathrm{p}<0.001)$.

\begin{tabular}{|c|c|c|c|}
\hline BANDS & COHERENCES & $p$ & MEANS \\
\hline \multirow[t]{3}{*}{ Delta } & Fpz-P8 & 0.007407 & $3>1$ \\
\hline & F7-T7 & 0.002472 & $3>1$ \\
\hline & F8-O1 & 0.004266 & $3>1$ \\
\hline \multirow[t]{5}{*}{ Theta } & Fp1-C3 & 0.005284 & $3>1$ \\
\hline & Fp1-P7 & 0.006338 & $3>1$ \\
\hline & Fp1-Pz & 0.001539 & $3>1$ \\
\hline & Fp2-C3 & $0.000288^{*}$ & $3>1$ \\
\hline & Fpz-C3 & 0.008132 & $3>1$ \\
\hline \multirow[t]{3}{*}{ Alpha } & F7-F8 & 0.007278 & $3>1$ \\
\hline & F7-T8 & 0.003511 & $3>1$ \\
\hline & T8-P7 & 0.007087 & $3>1$ \\
\hline \multirow[t]{23}{*}{ Beta } & Fp1-P3 & $0.000963^{*}$ & $3>1$ \\
\hline & $\mathrm{Fp} 1-\mathrm{Pz}$ & 0.002603 & $3>1$ \\
\hline & Fp1-P4 & 0.007310 & $3>1$ \\
\hline & Fp1-P8 & 0.005958 & $3>1$ \\
\hline & Fp1-O1 & 0.003563 & $3>1$ \\
\hline & FP1-O2 & 0.006766 & $3>1$ \\
\hline & Fp2-T7 & $0.000492^{*}$ & $3>1$ \\
\hline & $\mathrm{Fp} 2-\mathrm{Pz}$ & 0.007670 & $3>1$ \\
\hline & Fp2-O1 & 0.003080 & $3>1$ \\
\hline & Fpz-T7 & 0.008599 & $3>1$ \\
\hline & F7-C4 & 0.004885 & $3>1$ \\
\hline & F7-P3 & 0.002146 & $3>1$ \\
\hline & F7-Pz & 0.003526 & $3>1$ \\
\hline & F7-P4 & 0.001689 & $3>1$ \\
\hline & F7-O2 & 0.005344 & $3>1$ \\
\hline & F3-P3 & 0.001581 & $3>1$ \\
\hline & F3-Pz & 0.004113 & $3>1$ \\
\hline & Fz-T7 & $0.000498^{*}$ & $3>1$ \\
\hline & F4-T7 & 0.009187 & $3>1$ \\
\hline & F8-T7 & $0.000562^{*}$ & $3>1$ \\
\hline & T7-C4 & 0.006135 & $3>1$ \\
\hline & T7-T8 & 0.004112 & $3>1$ \\
\hline & T7-P7 & 0.006319 & $3>1$ \\
\hline
\end{tabular}

Table 2. Significant differences in coherence values between negative (2) and neutral (3) picture presentations. (Asterisk marks significance level <0.001).

\begin{tabular}{cccc}
\hline BANDS & COHERENCES & $\mathrm{p}$ & MEANS \\
\hline Delta & F8-Cz & $0.000773^{*}$ & $3>2$ \\
Theta & F4-C3 & 0.003370 & $3>2$ \\
Alpha & F7-F8 & 0.007425 & $3>2$ \\
& Fz-T8 & 0.001119 & $3>2$ \\
& F8-P7 & 0.004915 & $3>2$ \\
& T7-P8 & 0.008359 & $3>2$ \\
& Cz-O2 & 0.003932 & $3>2$ \\
& T8-P7 & 0.003020 & $3>2$ \\
T8-O1 & P3-O2 & 0.001434 & $3>2$ \\
\hline
\end{tabular}


can suppose that different processes could be represented by theta coherences. Sarnthein et al. [17] and Jensen \& Lisman [18] postulated that the theta frequency band in human EEG reflects memory processes. Chorlian et al. [19] also observed that theta coherent activity involved distinct midline and temporal sources (the same in our case) showing anterior/posterior differentiation. We may assume that in our case, theta coherences mark the recognition of the emotional valence of the stimuli.

Comparing our significant differences by emotional conditions the following findings were obtained:

Positive (1) and negative (2) pictures evoked very modest differences and at lower significance level than required for this study, consequently our data does not permit establish the strategies that brain uses in order to distinguish between the two types of stimuli. At the same time we can confirm, that there were no hemispheric differences according to valence. These results confirm the hypothesis and are in accordance with those reported in the meta-analysis of Wager et al. [13] In the same time, they are in open contradiction with the theory of Davidson [7] proposing right frontal activation to negative and left frontal activation for positive stimuli.

Distinguish between positive (1) and neutral (3) images was the most complex task, where subjects committed more mistakes, as neutral pictures are easily confounded with positive ones, resulting in significantly increased coherence values in the neutral (control) situation. Our results may reflect this increased resource using manifested by higher coherences in neutral condition, especially in the beta band. According to Petsche \& Etlinger [20] beta coherences are sensitive to mental effort that possibly emerges from a coordinated strong co-operation of these cell assemblies. In our study, this functional coupling occurred mostly between specific frontal, temporal and posterior interhemispheric areas.

When comparing neutral (3) and negative (2) images, we have obtained more significant differences in the alpha band. According to Cantero et al. [21] an increase in alpha coherence reflects changes in arousal level having maximum values in relaxed states. It is possible that neutral pictures, in spite of the difficulty in identification, are promoting a more relaxed state than negative pictures, then this observation could explain why we found higher alpha coherences for neutral pictures.

The inconsistency of findings in emotion research worth a mention, too. One possible reason for this is that although most studies assume that emotion-related activation patterns will be similar across people, there may be important individual differences in the biological basis of emotion and variation in the interpretation of affective images due to personality [22]. In the same manner, methodological differences do not make easier to get a final resolution [13].

\section{CONCLUSION}

EEG coherences do not distinguish between the emotional valences of the stimuli; rather they are internal indicators of different mental processes such as memory, mental effort and arousal. Our findings do not support the theory of hemispheric specialization, neither the theory of right hemisphere; they reveal a complex activation pattern that involves mostly frontal areas in connection with the entire cortex.

\section{ACKNOWLEDGEMENTS}

Our grateful thanks to the University of Florida for the permission of using the International Affective Pictures (Number, 2006. 1-16).

\section{REFERENCES}

[1] Ruchkin, D. (2005) EEG coherence. International Journal of Psychophysiology, 57, 83-85. doi:10.1016/j.ijpsycho.2005.04.001

[2] Schmid, R.G., Tirsch W.S., Rappelsberger, P., Weinmann, H.M. and Poppl, S.J. (1992) Comparative coherence studies in healthy volunteers and down's syndrome patients from childhood to adult age. Electroencephalography and Clinical Neurophysiology, 83, 112-123. doi:10.1016/0013-4694(92)90024-C

[3] Lopes da Silva, F.N. (1991) Neural mechanisms underlying brain waves: From neural membranes to networks. Electroencephalography and Clinical Neurophysiology, 79, 81-93. doi:10.1016/0013-4694(91)90044-5

[4] Bressler, S.L., Coppola, R. and Nakamura, R. (1993) Episodic multiregional cortical coherence at multiple frequencies during visual task performance. Nature, 366, 153-156. doi:10.1038/366153a0

[5] Andrew, C. and Pfurtscheller, G. (1996) Dependence of coherence measurement on EEG derivation type. Medical \& Biological Engineering \& Computing, 34, 232-238. doi:10.1007/BF02520079

[6] Russell, J.A. (1980) A circumplex model of emotion. Journal of Personality and Social Psychology, 39, 11611178. doi:10.1037/h0077714

[7] Davidson, R.J., Ekman, P., Saron, C., Senulis, J. and Friesen, W.V. (1990) Emotional expression and brain physiology I: Approach/withdrawal and cerebral asymmetry. Journal of Personality and Social Psychology, 58, 330-341. doi:10.1037/0022-3514.58.2.330

[8] Bos, D.O. (2006) EEG-based emotion recognition. The influence of Visual and Auditory Stimuli.

http://hmi.ewi.utwente.nl/verslagen/capitaselecta/CS-Oud e_Bos-Danny.pdf.

[9] Miskovic, V. and Miskovic, V. (2010) Cross-regional cortical synchronization during affective image viewing. Brain Research, 1362, 102-111. doi:10.1016/j.brainres.2010.09.102

[10] Güntekin, B. and Basar, E. (2010) Event-related beta oscillations are affected by emotional eliciting stimuli. Neuro- 
science Letters, 483, 173-178. doi:10.1016/j.neulet.2010.08.002

[11] Putman, P. (2011) Resting state EEG delta-beta coherence in relation to anxiety, behavioral inhibition, and selective attentional processing of threatening stimuli. International Journal of Psychophysiology, 80, 63-68. doi:10.1016/j.ijpsycho.2011.01.011

[12] Sutton, S.K., Davidson, R.J., Donzella, B., Irwin, W. and Dottl, D.A. (1997) Manipulating affective state using extended picture presentation. Psychophysiology, 34, 217226. doi:10.1111/j.1469-8986.1997.tb02135.x

[13] Wager, T., Phan, K.L., Liberzon, I. and Taylor, S.F. (2003) Functional neuroanatomy of emotion: A metaanalysis of emotion activation studies in PET and fMRI. Neuro Image, 16, 331-348.

[14] Volf, N.V. and Razumnikova, O.M. (1999) Sex differences in EEG coherence during a verbal memory task in normal adults. International Journal of Psychophysiology, 34, 113-122. doi:10.1016/S0167-8760(99)00067-7

[15] Clarke, A.R., Barry, R.J., McCarthy, R. and Selikowicz, M. (2001) Age and sex effects in the EEG: Development of the normal child. Clinical Neurophysiology, 112, 806814. doi:10.1016/S1388-2457(01)00488-6

[16] Briére, M.E., Foress, G., Chouinard, S. and Godbout, R. (2003) Evening and morning EEG differences between young men and women adults. Brain and Cognition, 53, 145-148. doi:10.1016/S0278-2626(03)00097-6

[17] Sarnthein, J., Petsche, H., Rappelsberger, P., Shaw, G.L. and von Stein, A. (1998) Synchronization between prefrontal and posterior association cortex during human working memory. Proceedings of the National Academy of Sciences, USA, 95, 7092-7096. doi:10.1073/pnas.95.12.7092

[18] Jensen, O. and Lisman, J.E. (1998) An oscillatory shortterm memory buffer model can account for data on the Sternberg task. The Journal of Neuroscience, 18, 1068810699.

[19] Chorlian, D.B., Rangaswamy, A.E. and Porjesz, B. (2009) EEG coherence: Topography and frequency structures. Experimental Brain Research, 198, 59-83. doi:10.1007/s00221-009-1936-9

[20] Petsche, H. and Etlinger, S.C. (1998) EEG aspects of cognitive processes: A contribution to the Proteus-like nature of consciousness. International Journal of Psychophysiology, 33, 199-212.

[21] Cantero, J.L., Atienza, M., Salas, R.M. and Gomez, C.M. (1999) Alpha EEG coherence in different brain status: An electrophysiological index $\mathrm{f}$ the arousal level in human subjects. Neuroscience Letters, 271, 167-170. doi:10.1016/S0304-3940(99)00565-0

[22] Canli, T., Zhao, Z., Desmond, J.E., Kange, E., Gross, J. and Gabrielli, J.D.E. (2001) An fMRI study of Personality influences on brain reactivity to emotional stimuli. Behavioral Neuroscience, 115, 33-42. doi:10.1037/0735-7044.115.1.33 